both the causes and the results of the accident. The utmost conceivable depth of "l,wness" is reached by a patient who, as a consequence of swallowing a corrosive acid suicidally, is for a long period neither able to pass food through the œsophagus nor chyme through the pylorus.

\section{The Question of TreatTMent.}

These cases seem to me to teach certain lessons. Conclusions should be drawn cautiously from a short series of only three cases; but the rules which $I$ am going to lay down are indicated by these cases, not only collectively, but individually. It is not, therefore, a mere matter of statistics.

1. The patient should receive no food (either liquid or solid) by the mouth for several weeks-i.e., he should not be fed by the mouth as soon as he can swallow with little or no pain; but oral feeding should be postponed until there is good reason to believe that the injuries have completely healed.

2. When the injuries are serious (and they generally are so) an operation should be performed within a few days of the date of the poisoning-the sooner the better

It must always be a matter of conjecture to determine whether the injuries have healed or not. The only parts of the injured tract visible are the mouth and pharynx, but the pylorus or even the middle of the stomach may be much worse, as, e.g., in Case 3, not to mention the oesophagus. In Case 3 the mouth and lips are noted as having healed on the eighteenth day, but active ulceration of the gullet was found after death on the eighty-fourth day (three months after the accident).

I am afraid that it is rarely safe to assume that a case of this kind is not serious unless it is positively known that only a minute quantity of acid has been swallowed. Case 3 was scarcely a truly suicidal one. The patient got drunk on the "rent money," was scolded by her husband, drank the acid, but spat most of it out again; she was brought to the hospital and made to swallow calcined lime and an emetic. and yet her injuries were terrible. Even when swallowed by pure accident an ounce or more is easily taken into the gullet before the mistake is discovered and most of it passes into the stomach,. running along the lesser curvature till it is stopped by the pylorus or by food already in the stomach.

All the three cases were treated according to what seems to be the usual practice-that is, the patients were allowed to swallow food when they could do it without much diffculty or pain. The patient in Case 2 was fed by the mouth from the very first day. The subject of Case 3 began with milk on the sixth day and took puddings on the fifteenth day. She never got as far as fish or meat. Granting that the patient should not be fed at all by the mouth for several weeks, and that we should only be satisfied with rectal feeding in trivial cases, the severe cases remain to raise the question of operative treatment.

The region most seriously injured is usually the pyloric part of the stomach. The problem for the surgeon's solution is not simple. The choice of operations apparently lies between gastrostomy, duodenostomy, jejunostomy, gastroenterostomy, and a combination of gastrostomy with gastroenterostomy. Gastrostomy does not give rest to the most injured part-namely, the pylorus. Gastro-enterostomy does not rest the cesophagus. Jejunostomy, when properly done, is practically a double operation. Duodenostomy would seem to be the simplest and most straightforward procedure, although it is liable to permit bile to leak out and irritate the skin. All these methods are open to the objection that, in the by no means unlikely event of an oesophageal or a pyloric contraction taking place after all, a secondary operation may have to be done, in addition to one for undoing the first operation. Therefore the indications would most likely be best met by combining a gastrostomy with a gastro-enterostomy, and carrying the gastrostomy tube through the gastro-enterostomy wound for some distance down the efferent loop of the jejunum. This is practically the method recommended by Witzel for an ordinary gastro-enterostomy, except that in the cases I am writing about something more is desirable-viz., an arrangement for washing out and draining the stomach through the gastrostomy wound. This could be obtained either by using a double tube, especially constructed so that the shorter canal opened into the stomach, or, more readily, by passing the long, narrow gastro-jejunal tube through a short, wide gastric tube. If in spite of treatment pyloric contraction should take place this plan provides a gastro-enterostomy ready made. The gastrostomy wound could be closed or kept open, according to the final condition of the cesophagus. In the hands of careful and experienced operators the method would probably be found very safe, as the patient would be operated on while in fair physical condition, and the gastro-jejunal tube should resist the dangers of the "vicious circle." 'These dangers could be further minimised by using Murphy's button, and, should that contrivance fall into the stomach, the gastrostomy wound itself could be enlarged to permit its extraction. Further, the treatment above recommended would reduce to a minimum the danger of infection of the air-passages, through the swallowing or the regurgitation of septic discharge, or of food, either of which might easily find its way through a glottis œdematous and stiffened either by direct injury or by injury to neighbouring parts. In a long series of cases of poisoning by corrosives now and then the glottis is likely to be so severely injured as to demand prompt tracheotomy. This would make it more than ever desirable not to feed by the mouth.

At the post-mortem examination of Case $3 \mathrm{I}$ was struck by the resemblance of the ulcerated bronchi to those of a case in which bronchial infection and gangrene had been caused by a tracheo-øesophageal fistula.

\section{AFTER-TREATMENT.}

Feeding by the gastro-jejunal tube would be commenced at once, in spite even of moderate ether or chloroform vomit ing, should those anæsthetics be used, but gas alone, or with oxygen, would suffice for the operation described, or even local anæsthesia. Local treatment should be given to (1) the mouth and nose, (2) the pharynx and the osophagus, and (3) the stomach

The mouth. - This should be frequently washed out with warm solution of chlorate of potash or warm boric lotion, or with both. Dirty teeth should be cleaned, diseased teeth should be treated with pure carbolic acid or by extraction, and suppurating alveoli should be attended to. If the process is very painful owing to the burning, gas, cocaine, or eucaine should be used. The nasal passages should be attended to if unhealthy; if healthy they should be left untouched.

The pharynx.-The pharynx is said not to be reached by gargles. It should be sprayed frequently with hot boric lotion, and twice a day it should be dusted with a little, not much, iodoform powder through a puff. As soon as the patient can swallow without pain he should be allowed hot water or hot neutral saline ad lib., and be encouraged to take it.

The stomach - The hot water swallowed should be allowed to escape by the short gastrostomy tube, so that it would tend to wash out the stomach also. In addition, after each meal given by the gastro-jejunal tube, the stomach should be washed out with hot water by the gastric tube.

The application in some such way as that above sketched of the principles of surgery to this distressing class of cases would, I believe, greatly reduce their mortality and lessen the permanent injury done to those who more or less recover Grosvenor-street, W.

\section{NOTES OF A SEVERE AND LONG-STAND- ING CASE OF LUPUS TREATED BY THE APPLICATION OF THE $\mathrm{X}$ RAYS.}

BY GEORGE H. RODMAN, M.D. DURH., M.R.C.S. ENG.

THE patient whose treatment is the subject of the following notes is a woman, aged about 33 years, who has suffered from lupus of the face, which is reported to have started after an operation for the removal of a diseased portion of the left malar bone some 20 years ago. There is a history of tuberculosis in the family, one sister having died from phthisis at the age of 14 years, and a brother also was the victim of laryngeal phthisis. The lupus had been scraped about 15 years ago; since then it has been treated medicinally and by the local application of ointments and lotions. At the time of starting the treatment by the $x$ rays, on April 19th, 1901, when Fig. 1 and Fig. 2 were obtained, the disease was involving both sides of the face, the ulceration being continued under the chin. On the left side of the face the disease extended from the zygomatic 
arch over the cheek and involved the lobe of the ear which the same time it caused frightful disfigurement and presented was considerably eroded from lupoid ulceration. Anteriorly the disease extended to within half an inch of the angle of the mouth. The upper portion of this area was covered with dry brownish scabs, the lower half being smoother and less ulcerated. The edges of the disease were considerably the features of typical lupus.

The x-ray treatment was commenced on April 19th, 1901. and between that date and July 29th, 1901, when it was

FIr. 3.

Fir: 1.

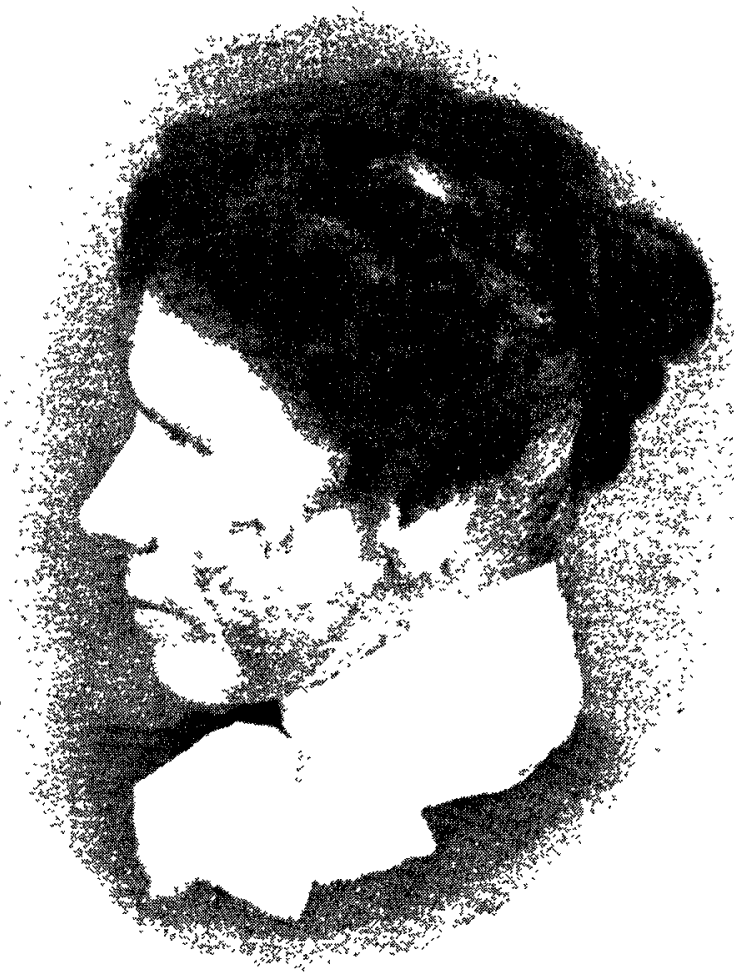

Showing the extent of the disease on the left side of the face on April 19th, 1901.

Frc: 2.

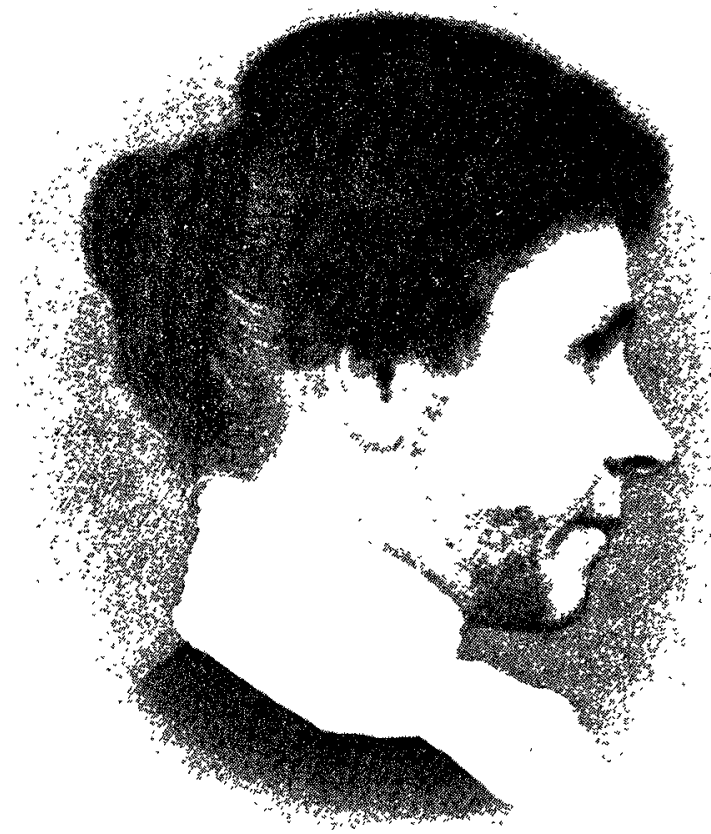

Showing the extent of the disease on the right side of the face on April 19th, 1901

raised and indurated. Below the jaw the disease consisted of a strip of abont an inch in width extending from the ramus of one side to that of the other; the scabbing was chiefly about the edges. On the right side of the face the disease was slightly less extensive than on the left, but at

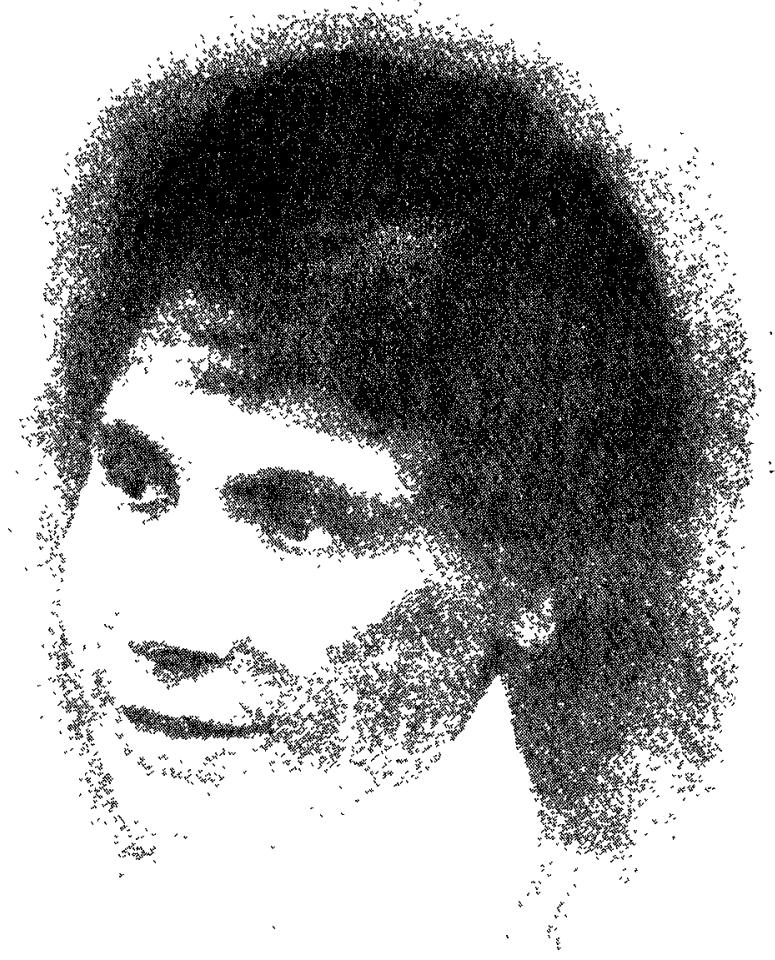

Showing the condition of the patient, left side, on Oct. 6th, 1901.

EIG. 4.

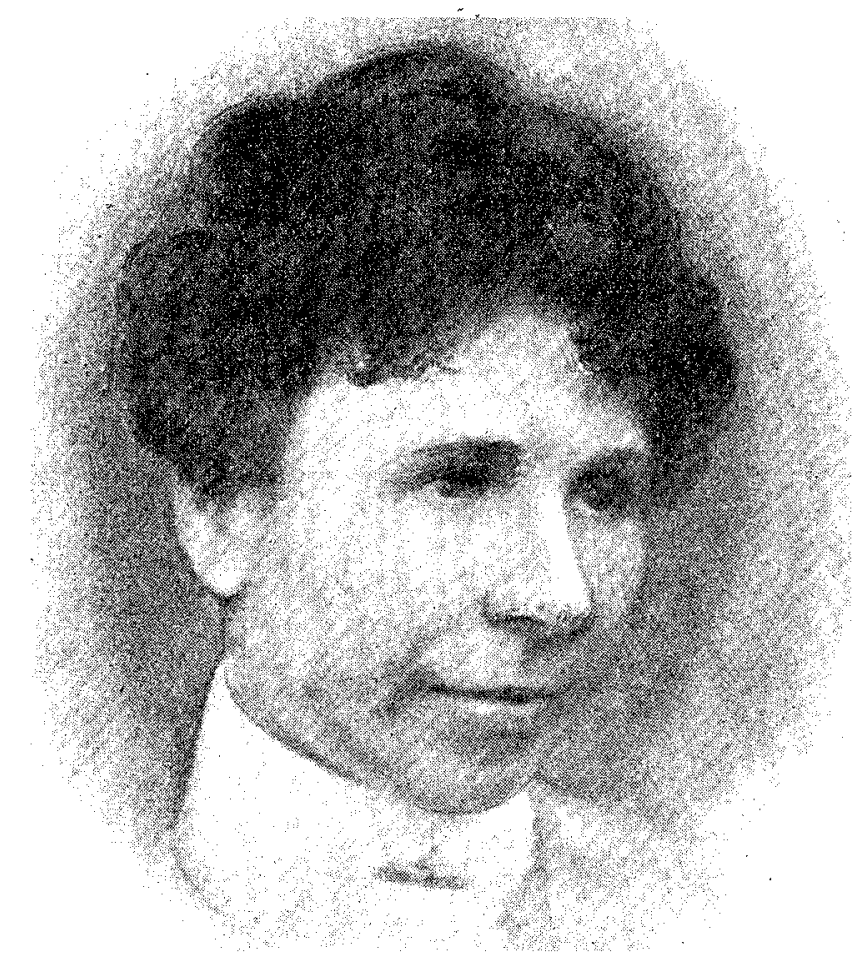

Showing the condition of the patient, right sicle, on Oct. 6th, 1901.

stopped, there were 50 exposures made, of which details are given in the accompanying table. 14 were employed in 
dealing with the left side on the first occasion. 17 were used to the right side, and on the second occasion of dealing with the left side there were 19 exposures made. On Sept. 13th, 1901, the healing process was complete with the exception of a small patch on the lobule of the left ear which it is my intention to expose again on some future occasion to the influence of the vacuum tube.

During the early sittings it will be noticed that the length of the exposures was shorter and the distance of the tube from the disease greater than that employed later-a course that should be invariably followed till the tolerance of the patient has been ascertained. Six applications were employed weekly during the period of active treatment.

The $x$ rays were produced, as will be seen from the table, with a potential of about eleven and a half volts, and the ampèrage employed was between two and three ampères in the primary current.

The tube used was a Cox's record tube excited by a 10-inch coil with platinum break. The portions of the face and neck not affected by the disease and the scalp were protected from the influence of the rays by a paper pulp mask, the outer surface of which was covered with a layer of thick lead foil. No falling off of the hair of the head or the eyebrows was noticed. Fig. 3 and Fig. 4 were obtained on Oct. 6th, 1901. These, as well as those before treatment was commenced, are from unretouched negatives kindly taken for me by my friend Mr. Albert Cheese. In Fig. 3 the dark discolouration of the left cheek over the seat of lupus has been exaggerated in the photograph.

Table giving Particulars of a Case of Lupus treated by the $X$ Rays.

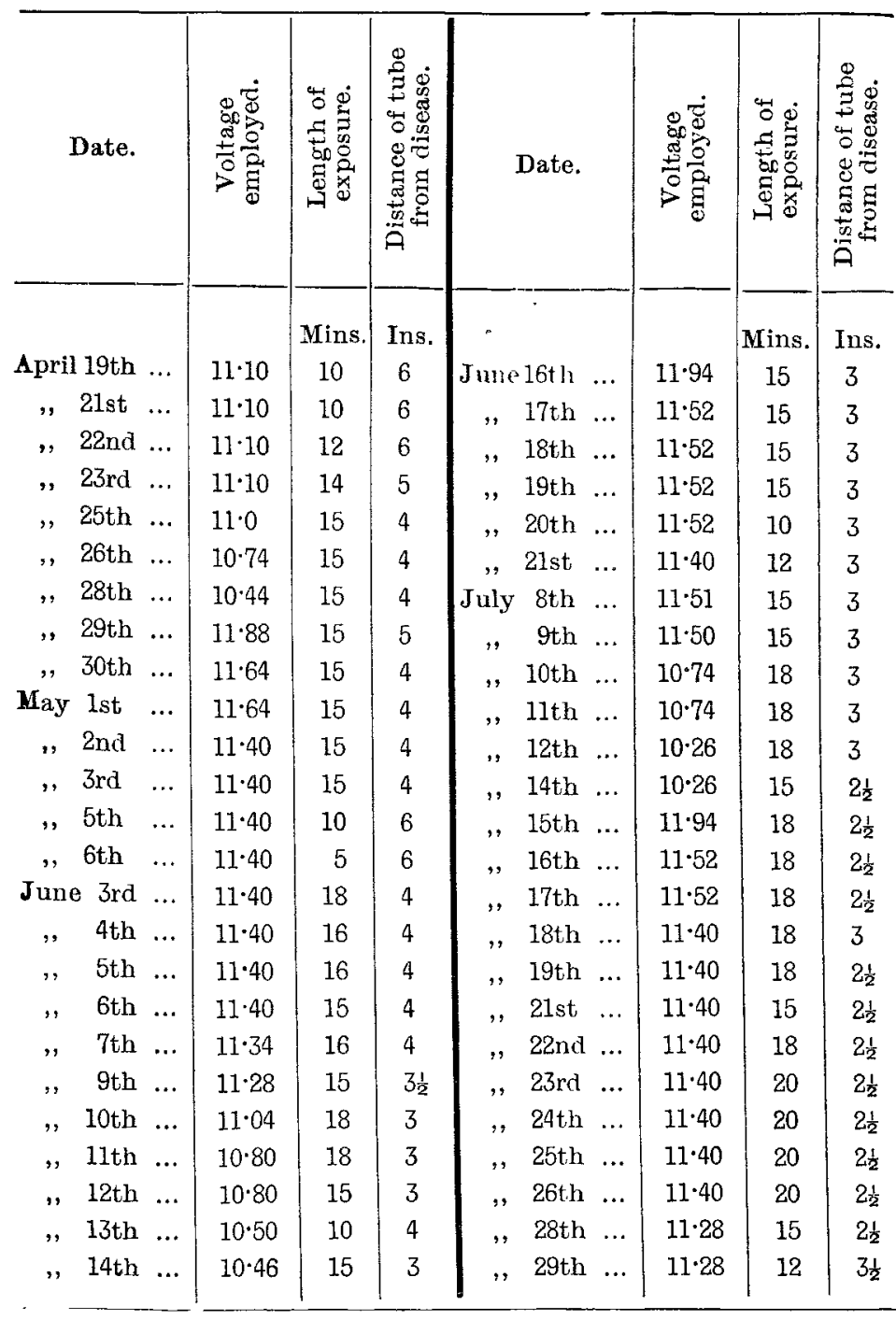

REMarks.

April 19th.-The application of the $x$ rays to the left side of the face was commenced.

April 28th. - The lobule of the ear was showing signs of irritation and was becoming raw from separation of scabs, consequently it was protected with lead-foil.

April $29 \mathrm{th}$. - The whole surface of the left side of the face was becoming redder.

May 1st.-Discharging pus in several points.

May 5th.- The patient complained of the surface being very tender. The lupus nodules at the upper and front edge were suppurating. The whole area looked flatter and there was not nearly so much infiltration of the erges.

May 6th. - The surface was discharging pus freely and the crust were separating; it was very tender and sore. The application of the rays was discontinued.

May 11th.-The crusts harl como away, leaving a clean, suppurating, aw, smooth surface resembling a hurn in process of healing.

May 2lst.-CThere was a white edge of healing ulceration round the raw surface. This enge varied in with $\mathrm{h}$ from $(\mathrm{n}$--eighth to one-quarter of an inch. In several spots there were islands of white healing centres.

May 26th. - The healing process proceeded rapidly, the white edge increasing in wilth. There were some remains of tubercular nodules at the extreme front.

June 2 nd.-Continued to heal rapidily.

June 3rd.- Treatment, was started on the right side of the face.

June 5th.- No reaction was noticed.

June 6th.-There was commencing hyperæmia of the ulcerated surface.

June 10th.-The healing process on the left side continued to progress atisfactorily.

June 16th.-The patient complained of the right cheek becoming sore. The surface began to look glazed. The healing of the left side f piece. patch of ulceration in the centre of the size of a threepenny-

June 20th.-Tenderness was more marked and suppuration was

June 21st.-The crusts had separated and the surface was freely uppurating. The application of the $\mathrm{x}$ rays was stopped.

June 25th. - The surface of the right cheek resembled that of a healing burn, the whole of the lupoid tissue having suppurated.

July 6th.- The surface had continued to suppurate freely and was now healing with a typical white edge extending over a smooth red raw surface.

Jnly 8th.-Treatment was reapplied to the left side of the face; a mask of lead-foil was applied over the parts cured and the rays were only allowed to invade the front edge of the scar, a patch over the zygoma, and the lobule of the ear.

July 12th. - The lobule of the ear was becoming slightly tender.

July 15th.-The ear was still tenderer, but at present there was no appearance of swelling of the lupoid erge on the cheek.

July 18th.- The ear was discharging freely, and consequently it was masked with lead foil.

July 22nd.-The ear was discharging freely; the edge of the scar on the cheek was becoming raised and tender

July 25 th.-Crusts were forming and becoming raised and the disJuly 25 th.-Crusts were forming
charging ear continued very tender.

July 28th.-The crusts were commencing to separate.

July 29 th. - The edge had become raw and suppurating. Application of the $\mathrm{x}$ rays was discontinued.

Sept. 13th. - The patient was completely healed.

East Sheen, S.W.

\section{A CASE OF LUPUS VULGARIS TREATED BY EXPOSURE TO X RAYS.}

\section{BY T. COKE SQUA N CE, M.D. DURH.,}

PHYSICLAN AND PATHOLOGIST TO THE SUNDERLAND INFIRMARY; ORAT EXAMINER IN PUBLIC HEALTH IN THE UNIVERSTTY OF DURHAM COLLEGE OF MEDICINE, NEWCASTLE-ON-TYNE.

ON July 15th, 1900, I was consulted by a woman with regard to her daughter, aged 17 years, who had suffered from lupus vulgaris for upwards of two years, during which period she had been on two occasions treated by erasion but without any permanent benefit, the disease having returned with increased virulency. Fig. 1 gives a very good idea of the appearance of the patient. Her nose was very much thickened and the alæ were ulcerating. The upper lip was extremely swollen, measuring a little over an inch from the skin to the mucous membrane. The disease extended over both cheeks, affecting the lower lids of both eyes. There was a patch on the forehead (concealed by the hair) and a large one extending over the angle of the jaw on the right side. I advised, as other methods had failed, that exposure to the $\mathrm{x}$ rays should be tried. This was agreed to, and at first I gave the patient 10 minutes' exposure three times a week, protecting the eyes as far as possible but allowing the lower lids to be exposed. After the first exposure there was decided flushing, accompanied by some pain and tingling and a sensation of heat which continued more or less markedly after each sitting, the skin assuming a reddishbrown appearance with yellowish crusts. As soon as the installation of the $\mathrm{x}$ ray apparatus at the Sunderland Infirmary was effected she became an inmate of the infirmary, and the subsequent exposures to the rays were made by $M r$. S. S. Larcombe (who had charge of the apparatus). For some time the exposures were made daily until the reaction became so marked that the treatment had to be intermitted, and the eyes also required attention, corneitis having supervened. Improvement was steady but slow until the condition shown in Fig. 2 was achieved, when the patient was practically cured, but as a precautionary measure occasional 